\title{
Inferences about eye movement control from the perceptual span in reading
}

\author{
ALEXANDER POLLATSEK and KEITH RAYNER \\ University of Massachusetts, Amherst, Massachusetts
}

and

DAVID A. BALOTA

Washington University, St. Louis, Missouri

\begin{abstract}
Subjects' eye fixations in a target region were examined as a function of how far the eye was from the beginning of a target word on the previous fixation. Prior to fixation of the target word, a letter string in the target location (which was then in the parafovea) was either visually similar or visually dissimilar to the target word. The results indicated that letter information was extracted from the target location when the eyes were at least nine characters from it. However, at such far distances, the parafoveal information extracted affected only the gaze duration on the target word, whereas at nearer distances, it affected both the gaze duration and the firstfixation duration. These data indicated that the decision to adjust the length of a saccade can be made after the decision to move the eye. In addition, the lengths of saccades and times to examine words were positively correlated: a longer saccade onto the target word was associated with longer gaze durations on both the target word and the prior word, and skipping the target word was associated with a longer last fixation on the previous word.
\end{abstract}

A great deal of recent research has indicated that the perceptual span, or the region from which we obtain useful information during eye fixations, in reading is limited (Den Buurman, Boersema, \& Gerrissen, 1981; Ikeda \& Saida, 1978; McConkie \& Rayner, 1975; Rayner \& Bertera, 1979; Rayner, Inhoff, Morrison, Slowiaczek, \& Bertera, 1981; Rayner, Well, \& Pollatsek, 1980; Rayner, Well, Pollatsek, \& Bertera, 1982; Underwood \& McConkie, 1985). The results of these experiments indicate that readers of English obtain useful information from a region extending 3 or 4 character positions to the left of fixation to approximately 15 character positions to the right of fixation (Rayner, 1984). Furthermore, within the perceptual span, different types of information are acquired. That is, information used for word or letter identification (Underwood \& McConkie, 1985) is obtained from a smaller region than is information used to determine where to look next (Pollatsek \& Rayner, 1982). The data indicate that information out to 14-15 character spaces is used to determine where to look next (McConkie \& Rayner, 1975; Rayner et al., 1981), whereas letter in-

\footnotetext{
This research represents a totally collaborative effort, and the order of the authors is random. The study was conducted while the third author held an NIMH postdoctoral fellowship at the University of Massachusetts, and the research was supported by Grants HD12727 and HD17246 from the National Institute of Child Health and Human Development. Thanks are extended to Peter DeGraef for his help with the data analysis and to Chuck Clifton, Albrecht Inhoff, and Kevin O'Regan for their helpful comments. Requests for reprints should be sent to Alexander Pollatsek at the Department of Psychology, University of Massachusetts, Amherst, MA 01003.
}

formation may only be acquired up to 8 characters from fixation (Underwood \& McConkie, 1985).

A related issue is how far from fixation lexical access can occur. The finding that highly predictable words are skipped more than are less predictable words (Balota, Pollatsek, \& Rayner, 1985; Ehrlich \& Rayner, 1981; O'Regan, 1979) indicates that lexical access of the word to the right of the currently fixated word can occur on that fixation (and, moreover, can influence the movement of the eye on the subsequent saccade). When the word to the right of the fixated word is identified on a fixation, the fixated word has undoubtedly been identified as well. However, the fixated word may not be fully processed on all fixations, since some words are fixated a second time.

This study had three focuses. The first was to explore more fully the question of how far into the parafovea useful letter information can be extracted. The second was to explore more fully the time course of information processing in reading, especially as it relates to control of eye movements. The third, which is an aspect of the second, was to determine whether there is a relationship between the length of saccades in reading and the length of time spent fixating words both immediately before and immediately after those saccades.

The last issue was of special interest partly because previous analyses had implied that the answer was negative. Rayner and McConkie (1976) analyzed a large number of reading protocols to determine the relationship between the duration of a fixation and the lengths of the saccades preceding and following it. They found these 
measures to be independent when averaged over the reading of an entire passage of text (see also Andriessen \& de Voogd, 1973; McConkie \& Zola, 1984). They interpreted these results as indicating that fixation duration and saccade length are controlled by separate mechanisms. Such independence could also be interpreted as suggesting that the moment-to-moment variation of fixation duration and saccade length is essentially random. However, in such an analysis, it is possible that there are both positive and negative correlations between the variables in different situations which average out to independence across the whole text. It is also possible that the duration of a single fixation is not the most sensitive measure to use. (We will discuss alternative measures below.) The present experiment allowed us to concentrate on a particularly informative part of the text to see whether there is a relationship between successive measures of the eye movement record.

The basic technique employed was the "boundary" technique used previously by Rayner (1975), in which subjects were asked to read a sentence in which an invisible boundary was set by the experimenter. (In the present experiment, the location of the boundary was 2 letters before the space preceding the target word.) $\mathrm{Be}$ fore the eyes crossed the boundary set by the experimenter, the letters in a target-word location were often altered. The relationship of the letter information in the target-word location before the eyes crossed the boundary to the target word allows one to make inferences about the kind of information extracted from the parafovea and its effect on the movement of the eyes.

The data reported here were collected as part of a study (Balota et al., 1985) in which we examined the interaction of the predictability of a target word and parafoveal information on the fixation time on the target word. In that study, we examined the fixation time on the target word independent of the distance of the prior fixation from the target word. In the present study, we focused on the effect of this distance on the acquisition of information, and, for the most part, ignored the effect of the predictability of the target word. We feel that averaging over different levels of predictability is reasonable, because, although the predictability of the target word interacted with several other variables, the analyses that we will discuss showed that the effects were obtained for both highly predictable and less predictable target words. Moreover, the design was completely factorial, so that predictability of the target word was counterbalanced in all the present analyses.

In the present study, we examined the first-fixation duration and the gaze duration on the target word as functions of (1) the parafoveal information available on the prior fixation and (2) the distance of the prior fixation point from the beginning of the target word. First-fixation duration is the duration of the first fixation on a word when there are multiple fixations and the duration of the single fixation when only one fixation on a word is made. Gaze duration represents the sum of all fixation time spent on a word prior to any eye movement away from it (Just \& Carpenter, 1980). Both measures were examined because of the possibility that they might reflect different components of processing (Balota et al., 1985; Inhoff, 1984). In addition to examining fixation time on the target word, we also examined fixation time on the word prior to fixation of the target to determine if there were any influences of the type of parafoveal information available.

\section{METHOD}

\section{Subjects}

Thirty members of the University of Massachusetts community were paid to participate in the experiment. All subjects had normal uncorrected vision and were naive with respect to the stimuli used in the experiment and with respect to the purpose of the experiment. Approximately half of the subjects had previously participated in an eye movement experiment.

\section{Procedure}

When a subject arrived for the experiment, a bite-bar was prepared that eliminated head movements. The initial calibration of the eye movement recording system was usually accomplished in less than $5 \mathrm{~min}$. This calibration was followed by 10 practice sentences and then the 100 experimental sentences. Before a sentence was presented, the experimenter ensured that calibration was correct and that the subject was fixating a cross near where the beginning of the sentence would be presented. The experimenter then gave a "ready" signal and presented the target sentence. After reading each sentence, the subject pressed a button which terminated the display. The subjects were instructed to read for comprehension, and were told that they would periodically be asked to release the bite-bar and report (verbatim or in a paraphrase) the sentence they had just read. They were also instructed to report if they noticed any abnormalities in the visual display (such as a display change) right after the sentence was presented.

\section{Materials and Apparatus}

Sentences extending over two lines (with up to 42 characters per line) were used as stimuli in the experiment. The target word could appear on either line, but was never at the beginning or end of either line. The target words ranged from 4 to 8 letters in length (mean length $=5.2$ letters), and the mean word frequency of the target words was 58 per million (KuCera \& Francis, 1967). Sentence frames were written such that two alternative words could appear in a critical word location. For example, in the sentence frame,

In her hair, the little girl wore a pretty pink __ that matched her dress;

the word ribbon or the word flower could be inserted in the critical word location. These two words each served as a target word in this frame: on half the trials, one word was present after the display change; on the other half of the trials, the alternative word was present. Although the two target words were always the same length, they were visually dissimilar. Table 1 presents some sample stimuli from the experiment.

Prior to the display change, one of five different letter strings could be present in the critical word location, exemplifying the five parafoveal conditions of the experiment. In the Ident (identical) condition, the target word was present before and after the display change. In the Rel (related) condition, one of the two target words was present before the reader's eye crossed the boundary location and then changed to the other target word after the reader's eye crossed the boundary (e.g., ribbon changed to flower or vice versa). In the VS (visually similar) condition, a nonword that was visually 
Table 1

Sample Stimuli in the Different Experimental Conditions

\begin{tabular}{lllll}
\hline \multicolumn{5}{c}{ Condition } \\
\hline Ident & Rel & VS & VD & Anom \\
\hline mouse & snake & mourc & snahc & paint \\
heart & liver & heanf & liven & files \\
clams & rocks & clars & rochs & signs \\
pans & sink & panz & sinh & doll \\
finger & pencil & finpen & penrnt & bakery \\
wallet & pocket & walhcf & pochcf & yellow \\
\hline
\end{tabular}

similar to the target was displayed before the eye crossed the boundary location (e.g., ribham changed to ribbon or flouon changed to flower). In the VD (dissimilar) condition, a nonword visually similar to the other target word, and hence visually dissimilar to the target word, preceded the target word (e.g., ribham changed to flower or flouon changed to ribbon). In the final condition, Anom (anomalous), a word that was totally unrelated to either of the word alternatives and which would make little sense in the sentence appeared prior to the display change (e.g., dreams would change to either ribbon or flower). All parafoveal previews were the same length as the target word.

Each subject received 20 sentences per condition. Each sentence frame occurred once in each of the five conditions. Hence, sentence frames were not repeated for any subject, and all sentences were ordered randomly in a unique fashion for each subject.

For each sentence, a boundary location was set two letter positions before the space to the left of the target word. Thus, in our example sentence, the boundary was located on the letter $n$ in pink. This boundary location was chosen to take advantage of where fixations usually fall on words (O'Regan, 1981; Rayner, 1979; Rayner \& McConkie, 1976). Readers do not often fixate on short words or on the end of a word; they tend to fixate on what has been called the preferred viewing location (Rayner, 1979), which is halfway between the beginning and the middle of a word. Thus, only a small percentage of saccades actually landed in the region between the boundary and the target word, since that region was always a short word or the end of a longer word. Within $5 \mathrm{msec}$ of when the reader's saccade crossed the boundary location, the initially presented stimulus was replaced by the target word.

Eye movement recording was accomplished by using a Stanford Research Institute Dual Purkinje eyetracker, which has a resolution of $10^{\prime}$ of arc and a linear output over the horizontal visual angle $\left(14^{\circ}\right)$ that was occupied by the sentences. The sentences were displayed on a Hewlett-Packard 1300A cathode ray tube (CRT), which has a P-31 phosphor with the characteristic that removing a character results in a drop to $1 \%$ of maximum brightness in $.25 \mathrm{msec}$. The sentences were printed with lowercase letters (except when capitals were appropriate). A black theater gel covered the CRT so that the letters appeared clear and sharp to the subjects. The eyetracker and the CRT were interfaced with a HewlettPackard 2100 computer which controlled the experiment. The signal from the eyetracker was sampled every millisecond, and the position of the eye was determined every $4 \mathrm{msec}$. Since the display change was accomplished within $5 \mathrm{msec}$ of when the eye actually crossed the boundary, the change occurred during the saccade (when vision is suppressed) in the vast majority of cases. The trials in which the display change occurred at the end of the saccade were eliminated (see the Results section).

The subject's eye was $46 \mathrm{~cm}$ from the CRT, and 3 characters equaled $1^{\circ}$ of visual angle. Although viewing was binocular, the right eye was monitored for eye movements. Luminance on the CRT was adjusted to a comfortable level for each subject, and the subjective brightness was held constant throughout the experiment. The room was dark, except for a dim indirect light source. More details about the characteristics of the apparatus are described in Rayner et al. (1981) and Rayner et al. (1982).

\section{RESULTS AND DISCUSSION}

The data discussed below are based on the mean per condition calculated for each of the 30 subjects. The only data that were removed from the analyses were the following: (1) sentences in which the eye-tracker lost track of the eye and, therefore, accurate measurements could not be made (6\%); (2) sentences in which subjects did not fixate the target word (5\%); (3) sentences in which the first fixation past the boundary landed on the two characters immediately to the left of the space before the target word (4\%). In such cases, the display change occurred before the target word was fixated. Also, there would be a greater chance for the subjects to have seen the display change occur, since the eye would have crossed the boundary as the saccade ended. In all cases in which the subjects reported that they had seen a display change (1\% of all trials), the saccade had crossed the boundary location at the end of the eye movement. Altogether, then, $15 \%$ of the trials were excluded from the analyses reported below. ${ }^{1}$

In a natural reading situation, of course, it is impossible to control experimentally where the reader fixates. Thus, we grouped fixations prior to boundary crossing into three categories: (1) near-3 to 5 character spaces from the beginning of the target word (with the space before the target word counting as the beginning of the target word); (2) middle-6 to 8 character spaces; and (3) far -9 or more character spaces. (Approximately $40 \%$ of the total observations fell into the near condition, $40 \%$ into the middle condition, and $20 \%$ into the far condition.) The analyses will be presented in the order in which the events occur as the reader moves to the target word.

\section{The Fixation Prior to Boundary Crossing}

The duration of the fixation prior to the boundary crossing was analyzed in order to assess how early various types of information affected the eye movement record. If semantic information was processed in the parafovea early enough in the fixation, then one might expect fixation duration prior to boundary crossing to be affected. For example, if subjects detected that the letters in the parafovea spelled a nonword, such as ribham or flouon given in our example, or a completely anomalous word, such as dreams, then one might expect the fixation duration prior to boundary crossing to be lengthened relative to the duration had a reasonable word been there. We will refer to the word fixated prior to the target word as Word $n-1$ (even though the word fixated was not always the one immediately preceding the target word).

Table 2 shows the duration of the last fixation and the gaze duration on Word $n-1$ for each of the five parafoveal preview conditions as a function of the distance of the last fixation on Word $n-1$ from the first letter of the target word. (For reasons that will be clear when we discuss the skipping data, this analysis excludes trials on which the target word was skipped.) There was no indication from the data that the presence of a semantic 
Table 2

Mean Fixation Duration (in Milliseconds) on the Fixation Immediately Prior to the Boundary Crossing and Mean Gaze Duration (in Milliseconds) for the Word Fixated Immediately Prior to the Boundary Crossing for the Five Experimental Conditions as a Function of Distance from the Beginning of the Critical Word Location

\begin{tabular}{|c|c|c|c|c|c|c|c|c|}
\hline \multirow[b]{3}{*}{ Condition } & \multicolumn{8}{|c|}{ Distance from Target } \\
\hline & \multicolumn{4}{|c|}{ Last-Fixation Duration } & \multicolumn{4}{|c|}{ Gaze Duration } \\
\hline & Near & Middle & Far & Mean & Near & Middle & Far & Mean \\
\hline Ident & 218 & 237 & 240 & 232 & 231 & 270 & 271 & 257 \\
\hline VS & 212 & 237 & 243 & 231 & 235 & 270 & 267 & 257 \\
\hline $\operatorname{Rel}$ & 227 & 229 & 249 & 235 & 240 & 261 & 261 & 254 \\
\hline Anom & 218 & 234 & 245 & 232 & 233 & 268 & 282 & 261 \\
\hline VD & 211 & 239 & 243 & 231 & 241 & 268 & 275 & 261 \\
\hline Mean & 217 & 235 & 244 & 232 & 236 & 268 & 273 & 259 \\
\hline
\end{tabular}

anomaly (either a nonword or an anomalous word) lengthened either the last fixation duration or gaze duration on Word $n-1$ (both $F \mathrm{~s}<1$ ). There was also no interaction of experimental condition with distance for either measure (again, both $F \mathrm{~s}<1$ ). There is thus no indication (from trials when the target word was not skipped) that the meaning of the parafoveal word (or anything else about it) had an effect on eye behavior prior to fixating the target word. Thus, the results are consistent with those reported previously by Rayner (1975) and Zola (1984). However, for the last fixation duration on Word $n-1$, there was a tendency for fixations to be longer when the reader fixated farther away from the beginning of the target word $[F(2,58)=2.11, p<.10]$, and the effect of gaze duration on Word $n-1$ was significant $[F(2,58)=$ $3.97, p<.05]$.

\section{Word Skipping}

The probability that the target word was skipped is a measure of whether it was processed in the parafovea. Since little information is extracted from the left of the fixated word (McConkie \& Rayner, 1976; Rayner, Well, \& Pollatsek, 1980), it seems reasonable to assume that the target word is identified during the fixation before it is skipped. The reasonableness of this assumption is greater when the probability of the occurrence of the word being skipped is related to some meaningful variable such as its predictability from the prior text. Such a result was found in prior experiments (Ehrlich \& Rayner, 1981; O'Regan, 1979) and was found in the current data (also see Balota et al., 1985). Although the sentences in which subjects skipped the target word were excluded from analyses involving fixation times on the target word, they were further analyzed to determine how far from the target word one could be and still skip the target word. Altogether, as mentioned above, subjects skipped over the target word $5 \%$ of the time. Of those trials on which the target was skipped, the saccade was initiated from the near region $65 \%$ of the time, from the middle region $27 \%$ of the time, and from the far region $8 \%$ of the time. Overall, the probability that the target would be skipped was .083 when the reader's fixation was in the near region,
.033 when it was in the middle region, and .015 when it was in the far region. Thus, for the most part, when readers skipped over the target location, they had fixated quite close to the target word. ${ }^{2}$

Another question of interest was whether the fixation duration on Word $n-1$ was affected by whether or not the target word was skipped. Our analysis showed that the mean duration of the last fixation was $21 \mathrm{msec}$ longer when the target word was subsequently skipped than when it was not $[t(19)=5.78, p<.01]$ and that gaze durations were $51 \mathrm{msec}$ longer $[t(19)=8.98, p<.001]{ }^{3}$ A similar result was reported by Hogaboam (1983). This suggests that identification of the parafoveal word can affect the current fixation duration. To guard against the possibility that the longer fixation durations were due to differences in the prior landing position, the data from the near condition were analyzed separately, and the same pattern of data obtained. (Skipping data in the middle and far conditions were insufficient for a meaningful analysis.)

\section{The First Fixation After Boundary Crossing}

We now turn our attention to the first fixation on the target word. As can be seen from Table 3, there was a marked effect of experimental condition $[F(4,116)=$ $4.22, p<.01]$. The main effect of distance $(p>.25)$ and the interaction of distance with parafoveal condition $(F<1)$ were not close to being significant. Since most of the difference was between conditions in which the parafoveal stimulus was visually similar to the target word (the Ident and VS conditions) and those in which it was visually different (the Rel, VD, and Anom conditions), we decided to focus our analysis on a contrast in which visually similar conditions were compared with visually dissimilar conditions at each of the three distances (see Table 4). The data reveal that when the parafoveal preview was visually similar to the target word, the firstfixation duration was $13 \mathrm{msec}$ shorter than when it was visually dissimilar $[F(2,58)=13.79, p<.001]$. Of greatest interest is that there was a significant interaction of distance with visual similarity $[F(2,58)=3.26$, $p<.05]$, with visual similarity having $21-, 16-$, and $3-$ msec effects on first-fixation duration when the prior fixation was 3-5, 6-8, or 9 or more characters, respectively, from the fixated word. The effect of visual similarity was

Table 3

Mean First-Fixation Duration (in Milliseconds) and Mean Gaze Duration (in Milliseconds) on the Target Word Following the Display Change for the Five Experimental Conditions as a Function of the Distance Between the Prior Fixation and the Beginning of the Critical Word Location

Distance from Target

\begin{tabular}{|c|c|c|c|c|c|c|c|c|}
\hline \multirow[b]{3}{*}{ Condition } & \multirow{2}{*}{\multicolumn{4}{|c|}{ n Duration }} & & & & \\
\hline & & & & & \multicolumn{4}{|c|}{ Gaze Duration } \\
\hline & Near & Middle & Far & Mean & Near & Middle & Far & Mean \\
\hline Ident & 219 & 220 & 224 & 221 & 241 & $24 \overline{7}$ & 264 & 250 \\
\hline VS & 226 & 224 & 227 & 226 & 250 & 257 & 269 & 25 \\
\hline Rel & 237 & 235 & 229 & 233 & 260 & 306 & 309 & 29 \\
\hline Anom & 249 & 243 & 227 & 240 & 271 & 308 & 296 & 292 \\
\hline VD & 244 & 235 & 228 & 236 & 270 & 284 & 308 & 287 \\
\hline Mean & 235 & 232 & 227 & 231 & 258 & 280 & 289 & 276 \\
\hline
\end{tabular}


Table 4

Mean First-Fixation Duration (in Milliseconds) for the Three Distances Comparing Similar (Ident and VS) and Dissimilar (Rel, Anom, and VD) Conditions

\begin{tabular}{|c|c|c|c|c|}
\hline \multirow[b]{2}{*}{ Condition } & \multicolumn{4}{|c|}{ Distance from Target } \\
\hline & Near & Middle & Far & Mean \\
\hline $\begin{array}{l}\text { Similar } \\
\text { Dissimilar }\end{array}$ & $\begin{array}{l}222(243) \\
243(266)\end{array}$ & $\begin{array}{l}221(252) \\
236(289)\end{array}$ & $\begin{array}{l}228(260) \\
229(303)\end{array}$ & $\begin{array}{l}224(252) \\
236(286)\end{array}$ \\
\hline Difference & $21 \quad(23)$ & $15 \quad(37)$ & $1 \quad(43)$ & $12(34)$ \\
\hline
\end{tabular}

Note-Values in parentheses are gaze durations in milliseconds.

significant at both the near and middle distances (both $p s<.01$ ), but was clearly not so for the far distance $(F<1)$. (There was no main effect of distance, however; $F<1$.)

Thus, when one looks at first-fixation duration, it appears that the visual similarity of the parafoveal information to the target word affected the fixation duration only if the prior fixation was 8 or fewer characters from the target word. This suggests that letter information was being extracted only out to about 8 characters to the right of fixation. A different picture emerges, however, when one looks at gaze duration.

\section{Gaze Duration}

The gaze duration data are presented in Tables 3 and 4. As with first-fixation duration, there was a strong effect of parafoveal preview condition $[F(4,116)=25.19$, $p<.001]$, and, as with first-fixation duration, most of the effect can be explained by a difference between visually similar and visually dissimilar previews $[F(1,29)$ $=65.21, p<.001]$.

The gaze duration data, however, differ from the firstfixation duration data in two ways. The first is that the distance of the previous fixation from the target word affects gaze duration $[F(2,58)=9.24, p<.001]$, with gaze duration going from $236 \mathrm{msec}$ in the near condition to $273 \mathrm{msec}$ in the far condition. Thus, the larger the saccade to the target word, the longer the time spent on it. The second is that the interaction between distance and visual similarity was not significant $[F(2,58)=1.42$, $p>.25]$ and, if anything, visual similarity had greater effects at the longer distances. In addition, the effect of visual similarity was significant at all three distances (all $p s<.001)$. The latter finding is important, since it implies that subjects were extracting letter information from further away than 8 characters from the fovea. However, visual similarity affected only gaze duration and not firstfixation duration at these longer distances. We will discuss the implications of the latter finding in the next section.

\section{CONCLUSIONS}

There are several important conclusions to be drawn from the present study. First, our results indicate that letter information can be extracted at least 9 character spaces from the fixation point, since the visual similarity of the parafoveal preview to the target word affected processing even when the fixation prior to the target-word fixa- tion was at least 9 character spaces away. ${ }^{4}$ However, the effect of processing that letter information (when it was so distant from the fixation point) appeared not in the duration of the first fixation on the target word, but in the gaze duration, which chiefly reflects the probability of fixating the target word a second time. Thus, if an analysis is done only on the duration of the first fixation on a word (e.g., Underwood \& McConkie, 1985), effects of letter information further away than 6-8 character spaces from the fixation point might be missed.

Second, our results support the finding (Rayner, 1975) that partial word information acquired parafoveally is useful on the next fixation. In our experiment, the VS condition preserved the first 2 or 3 letters of the target word, and we found little difference between it and the Ident condition (in which there was no display change). On the other hand, when the parafoveal preview was visually different from the target word, fixation time on the target word increased. Thus, our data are consistent with the view that parafoveal information acquired during the prior fixation influences processing on the subsequent fixation (see Balota et al., 1985).

Third, contrary to earlier analyses, our analyses indicated that fixation times and saccade lengths can be related: (1) the gaze duration on Word $n-1$ was longer when the saccade to the target word was longer; (2) the gaze duration on the target word was also longer when the saccade to it was longer; and (3) both the last fixation duration and gaze duration on Word $n-1$ were longer when the target word was skipped than when it was not. These three results all suggest that there are places in text where more complete processing of the material fixated (and that just to the right of fixation) takes more time but then leads to longer saccades. The prior findings (McConkie \& Zola, 1984; Rayner \& McConkie, 1976) of no relationship between saccade length and fixation duration, when averaged over a whole text, may be due in part to the likely occurrence of other places where longer fixations and short saccades go together, as when text processing is particularly difficult (see Frazier \& Rayner, 1982).

A causal relationship between long saccade lengths and long fixation times can only be suggested by the correlational methodology of the present study. Although it is difficult to develop an alternative explanation for why fixation time would be longer on Word $n-1$ when Word $n$ is skipped, the other two relationships may be subject to alternative interpretations. Longer gaze durations on Word $n-1$ when a longer saccade was made to Word $n$ may be due merely to the fact that the words fixated when readers were further away from the target position were different from those fixated when they were nearer. There is little in the present experiment that allows us to assess whether this distance effect is merely a function of the material fixated; however, the consistency of this effect with the obtained skipping data at least suggests that it is not totally due to materials.

Longer gaze durations on Word $n$ as a function of distance (see Table 3) may also have an alternative expla- 
nation. When the target word is approached from a farther distance, the location of the fixation point on the target word may be different from that found when it is approached from a shorter distance. The distance effect in gaze duration, therefore, may be due to the different firstfixation point on the target word. The first-fixation point could influence the gaze duration if the legibility of a word varies depending on where it is fixated. In reading, it has been shown that fixation points on words are not random; although there is considerable variability in where words are fixated, fixations tend to fall a bit to the left of the center of a word (Dunn-Rankin, 1978; O'Regan, 1981; Rayner, 1979). This pattern has suggested that these preferred viewing locations (Rayner, 1979) represent points of maximum legibility. This idea has been expanded by O'Regan, Levy-Schoen, Pynte, and Brugaillere (1984), who have demonstrated that when words in isolation are identified the probability of refixation and gaze duration increases dramatically when the initial fixation is to the left or right of an optimal location. (O'Regan et al. also pointed out that this optimal location may not be the same as the preferred viewing location, since the latter may be influenced by factors other than the legibility of the fixated word.)

To assess the possibility that our distance effects were primarily due to fixation location, we analyzed the data of 10 subjects selected at random from the 30 subjects to determine whether such an explanation was plausible. In fact, as shown in Table 5, subjects were more likely to land nearer the beginning of the target word in the far condition than in the near condition. Moreover, most of the effect in gaze duration was due to the probability that the word was fixated twice (see Table 5). Thus, there is a confounding: a longer saccade to the target word is related to a different landing location on the target word. Even so, one might be able to tentatively decide, on the basis of plausibility, among various explanations for why gaze durations are lengthened (and probability of refixation increased) when the target word is approached from a farther distance.

Table 5

Landing Position, Probability of Refixation, and Duration of Refixation for Similar (Ident and VS) and Dissimilar (Rel, Anom, and VD) Conditions as a Function of Distance from the Target Word for 10 Subjects

\begin{tabular}{llccc}
\hline Condition & $\begin{array}{c}\text { Distance } \\
\text { from Target }\end{array}$ & $\begin{array}{c}\text { Landing } \\
\text { Position* }\end{array}$ & $\begin{array}{c}\text { Probability } \\
\text { of Refixation }\end{array}$ & $\begin{array}{c}\text { Duration } \\
\text { of Refixation } \\
\text { (milliseconds) }\end{array}$ \\
\hline \multirow{3}{*}{ Similar } & Near & .49 & .11 & 168 \\
& Middle & .36 & .21 & 177 \\
& Far & .25 & .25 & 167 \\
\multirow{5}{*}{ Dissimilar } & Near & .49 & .20 & 169 \\
& Middle & .36 & .41 & 186 \\
& Far & .24 & .58 & 175 \\
\hline
\end{tabular}

*Value computed represents the mean proportion of the distance into the word at which the eye landed. For example, if the subject landed on the second letter of a 5-letter word, the value would equal .40 , while if the reader landed on the third letter of an 8-letter word, the value would be .375 , and so on.
The simplest, and least interesting, explanation is that the refixation of the word is due merely to the fact that a fixed percentage of saccades from any location are short; hence, a short saccade is more likely to fall on the same word when the prior fixation is near the beginning of the word than when the first fixation is farther into the word. This explanation can easily be dismissed, since gaze duration and the probability of refixation were both strongly affected by visual similarity. Thus, the probability of refixating the target word was due not only to where the reader first landed on a word, but also to the compatability between the parafoveal preview and the target word. However, it is still possible that refixations are at least partially due to poor visual information's accruing from the end of the word when the beginning is fixated. Here, again, the effect of visual similarity makes this less plausible. In the far condition, the end of the target word was always at least 13 characters from the fixation point on Word $n-1$, and so it is unlikely that the preview enhanced the perception of the end letters even in the Ident condition (McConkie \& Rayner, 1975; Rayner \& Bertera, 1979). Thus, if refixating the word in the far condition is chiefly due to poor visual information at the end of the target word, it seems unlikely that the visual preview is going to affect that. Rather, it seems more plausible that when fixation of Word $n-1$ is further from the target word, the reader is less far along in processing the information from the preview, and hence needs to spend more time on the target word.

The final point we wish to discuss is the fact that visual similarity had an effect on first-fixation duration of the target word at the near distance but had an effect on gaze duration (through the probability of refixating the word) only at the far distance (see Tables 4 and 5). There appear to be two possible explanations for this pattern of results. The first explanation, elaborated below, is that the decision of whether to refixate a word or not can be made later in the first fixation on a word than the decision of when to terminate that fixation. ${ }^{5}$ The second explanation is that the difference is due to the landing position: when the first fixation is on the beginning of a word, the decision to spend more time results in a refixation, whereas when the first fixation is made further into the word, the decision to spend more time results in a lengthening of the fixation. The latter explanation, while possible, relies on what appears to be a fairly complex judgment: the reader needs to compute the fact that he or she is fixated near the beginning of a long word and, on that basis, to decide to execute a refixation rather than a lengthening of the fixation when processing of the fixated word is delayed. In contrast, the first explanation relies only on positing a quantitative difference-that decisions of where to go can be made later than decisions of when to go.

The argument for why refixation decisions are made later than decisions about when to terminate the fixation is as follows. The visual similarity of the preview to the 
target word appears to influence the time needed to process the target word. Most of the effect is probably due to the fact that letter information has been extracted from the preview which aids lexical access (Balota et al., 1985; Rayner, McConkie, \& Zola, 1980). When the preview information is good (when fixation $n-1$ is near the target word), lexical access is rapid enough to affect the decision of when to move the eyes. However, when fixation $n-1$ is further from fixation, poorer preview information would be extracted and lexical access is likely to be slower. Thus, the most plausible explanation for the fact that first-fixation duration is not affected when fixation $n-1$ is at the far distance is that letter information extracted parafoveally from the target location does not speed lexical access sufficiently to be able to beat the decision to move the eyes. The fact that the letter information influences the probability of refixating the word at the far distance indicates that some letter information has been acquired which is unable to influence the decision to terminate the first fixation but is able to influence the later decision of where to fixate next. The same conclusion follows from an analysis of a different experiment.

Inhoff and Rayner (1986) varied both the frequency of a target word (holding the number of letters constant) and whether there was a parafoveal preview of the word. As in the present study, they measured both the mean firstfixation duration and mean gaze duration on the target word when it was fixated. Their findings were that word frequency affected both first-fixation duration and gaze duration when there was a parafoveal preview of the target word, but affected only gaze duration when there was no parafoveal preview. This pattern of data is easily explained by making the same two assumptions to explain the data of the present experiment: (1) lexical access is slower if there is poorer parafoveal information (in this case none); and (2) the decision to refixate can be made later than the decision of when to terminate the first fixation. Thus, when there is no parafoveal preview, lexical access times, even for the high-frequency words, are never fast enough to influence the decision to end the first fixation; however, the lexical access times for the highfrequency words are fast enough to influence the decision of whether to refixate the word and can affect gaze duration. On the other hand, when there is a parafoveal preview, lexical access times for high-frequency words are fast enough to influence both decisions and can therefore affect both measures.

Both experiments (the present one and Inhoff \& Rayner's) thus suggest that first-fixation duration on a word is unlikely to be affected by the time of lexical access unless there has been a healthy dose of parafoveal information acquired on the prior fixation. Accordingly, both experiments also suggest that gaze duration may in many cases be a more sensitive measure of processing than first-fixation duration, since the gaze duration may reflect processing events later in the first fixation than the duration of the first fixation. The argument also implies that the decision to move the eyes is made before lexical access is complete under conditions in which good parafoveal previews are not obtained. Further work is needed to determine whether the decision to move the eyes prior to lexical access is, in some cases, "automatic" and unaffected by ongoing cognitive processes or whether there are processing stages short of full lexical access that trigger the decision to move the eyes in reading.

\section{REFERENCES}

ANDRIESSEN, J. J., \& DE VoOGD, A. H. (1973). Analysis of eye movement patterns in silent reading. IPO Annual Progress Report, 8, 30-35. Balota, D. A., Pollatsek, A., \& Rayner, K. (1985). The interaction of contextual constraints and parafoveal visual information in reading. Cognitive Psychology, 17, 364-390.

Den Buurman, R., Boersema, T., \& Gerrissen, J. F. (1981). Eye movements and the perceptual span in reading. Reading Research Quarterly, 16, 227-235.

Dunn-RankIN, P. (1978). The visual characteristics of words. Scientific American, 238, 122-130.

EHRLich, S. F., \& RAYNER, K. (1981). Contextual effects on word perception and eye movements during reading. Joumal of Verbal Learning \& Verbal Behavior, 20, 641-655.

FRAZIER, L., \& RAYNER, K. (1982). Making and correcting errors during sentence comprehension: Eye movements in the analysis of structurally ambiguous sentences. Cognitive Psychology, 14, 178-210.

HogabonM, T. W. (1983). Reading patterns in eye movement data. In $\mathrm{K}$. Rayner (Ed.), Eye movements in reading: Perceptual and language processes (pp. 309-332). New York: Academic Press.

IKEDA, M., \& SAIDA, S. (1978). Span of recognition in reading. Vision Research, 18, 83-88.

INHOFF, A. W. (1984). Two stages of word processing during eye fixations in the reading of prose. Journal of Verbal Learning \& Verbal Behavior, 23, 612-624.

INHOFF, A. W., \& RAYNER, K. (in press). Parafoveal word processing during eye fixations in reading: Effects of word frequency. Perception \& Psychophysics.

Just, M. A., CARPENTER, P. A. (1980). A theory of reading: From eye fixations to comprehension. Psychological Review, 87, 329-354.

KuČrRA, H., \& Francis, W. N. (1967). Computational analysis of present-day American English. Providence, RI: Brown University Press.

McConkIE, G. W., \& RAYNer, K. (1975). The span of the effective stimulus during a fixation in reading. Perception \& Psychophysics, 17, 578-586.

MCCONKIE, G. W., \& RAYNer, K. (1976). Asymmetry of the perceptual span in reading. Bulletin of the Psychonomic Society, 8, 365-368.

McCoNXIE, G. W., ZolA, D. (1979). Is visual information integrated across successive fixations in reading? Perception \& Psychophysics, 25, 221-224.

McConkIE, G. W., \& ZoLA, D. (1984). Eye movement control during reading: The effect of word units. In W. Prinz \& A. F. Senders (Eds.), Cognition and motor processes (pp. 63-74). Berlin: Springer.

O'REGAN, J. K. (1979). Saccade size control in reading: Evidence for the linguistic control hypothesis. Perception \& Psychophysics, 25, $501-509$.

O'REGAN, J. K. (1981). The "convenient viewing position"' hypothesis. In D. F. Fisher, R. A. Monty, \& J. W. Senders (Eds.), Eye movements: Cognition and visual perception (pp. 289-298). Hillsdale, NJ: Erlbaum.

O'Regan, J. K., Levy-Schoen, A., Pynte, J., \& Brugaillere, B. (1984). Convenient fixation location within isolated words of different length and structures. Journal of Experimental Psychology: $\mathrm{Hu}$ man Perception \& Performance, 10, 250-257. 
Pollatsek, A., \& RAYNer, K. (1982). Eye movement control in reading: The role of word boundaries. Journal of Experimental Psychology: Human Perception \& Performance, 8, 817-833.

RAYNER, K. (1975). The perceptual span and peripheral cues in reading. Cognitive Psychology, 7, 65-81.

RAYNER, K. (1979). Eye guidance in reading: Fixation locations within words. Perception, 8, 21-30.

RAYNER, K. (1984). Visual selection in reading, picture perception, and visual search: A tutorial review. In H. Bouma \& D. G. Bouwhuis (Eds.), Attention and performance $X$ (pp. 67-96). Hillsdale, NJ: Erlbaum.

RAYNER, K., \& BERTERA, J. H. (1979). Reading without a fovea. Science, 206, 468-469.

RAYNer, K., INHOFF, A. W., MoRrison, R., SLowiaczeK, M., \& BeRTERA, J. (1981). Masking of foveal and parafoveal vision during eye fixations in reading. Journal of Experimental Psychology: Human Perception \& Performance, 7, 167-179.

RAYNER, K., \& McConkIE, G. W. (1976). What guides a reader's eye movements? Vision Research, 16, 829-837.

RAYNER, K., MCCONKIE, G. W., \& ZoLA, D. (1980). Integrating information across eye movements. Cognitive Psychology, 12, 206-226.

RaYner, K, \& Pollatsek, A. (1981). Eye movement control during reading: Evidence for direct control. Quarterly Joumal of Experimental Psychology, 33A, 351-373.

Rayner, K., Well, A. D., \& Pollatsek, A. (1980). Asymmetry of the effective visual field in reading. Perception \& Psychophysics, 27, 537-544.

Rayner, K., Well, A. D., Pollatsek, A., \& Bertera, J. H. (1982). The availability of useful information to the right of fixation during reading. Perception \& Psychophysics, 6, 537-550.

UNDERWOOD, N. R., \& McCoNXIE, G. (1985). Perceptual span for letter distinctions during reading. Reading Research Quarterly, 20, 153-162.

ZoLA, D. (1984). Redundancy and word perception during reading. Perception \& Psychophysics, 36, 277-284.

\section{NOTES}

1. Instances in which a reader's left-to-right saccade crossed the boundary immediately after the reader had made a regression were also ex- cluded from the data analysis. In such cases, the distance from the target word on the fixation prior to boundary crossing may not accurately reflect the parafoveal preview that had been obtained from the target word, since the reader's fixation was closer to the target word on the prior fixation. Such instances were quite rare in the data set, amounting to about $0.1 \%$ of all trials.

2. The probability of skipping is not merely a function of the distance from the target word, since it was strongly influenced by the predictability of the target word from the prior sentence context. Thus, skipping in the present experiment is a sensitive index of cognitive processing. For a fuller discussion of this point, see Balota et al. (1985).

3. The comparison of fixation durations conditional on skipping and on not skipping the target word was possible for only 20 of the subjects: 10 of the subjects skipped the target word so infrequently that there were not enough data to compute a reliable mean fixation time for when they skipped.

4. In this paper, we assume that the improved processing when the parafoveal preview is visually similar is due to extraction of letter information in the parafovea. This is based on much prior research. Prior experiments have demonstrated that a change of letter case from fixation to fixation had no effect on processing, and thus that visual features are probably not integrated from fixation to fixation (McConkie \& Zola, 1979; Rayner, McConkie, \& Zola, 1980). In addition, experiments have demonstrated that visual changes in the parafovea that do not affect letter information or word boundary information also have no effect on reading (e.g., Pollatsek \& Rayner, 1982).

5. We do not wish to imply that all decisions about where to move the eye are made late in the fixation. In fact, other data (Pollatsek \& Rayner, 1982; Rayner \& Pollatsek, 1981) suggest that at least a preliminary decision concerning where to move the eyes next is made on the basis of visual information that appears within approximately the first $50 \mathrm{msec}$ of a fixation and is based on the location of word boundaries to the right of fixation.
(Manuscript received January 13, 1986; revision accepted for publicaton June 11, 1986.) 